example, the $\mathrm{J} / \psi$ at $3.1 \mathrm{GeV}$ and the upsilon at $9.4 \mathrm{GeV}$. In these bound states the heavy quark-antiquark pair can decay hadronically only by annihilating into three gluons in much the same way that positronium disintegrates into three photons. The gluons of course have to evolve into colourless hadrons later. The glueball may lurk among such hadrons. One interesting decay mode is when $\mathrm{J} / \psi$ goes into an energetic photon plus hadrons. Here the heavy quark-antiquark pair in the $\mathrm{J} / \psi$ annihilate into two gluons and a photon. The two gluons could resonate into a glueball in which case the photon will be highly monochromatic. Experiments done by the PLUTO group at the German storage ring DORIS have yielded good quality data for precisely this situation. They rule out the existence of any glueball up to a mass of $1.2 \mathrm{GeV}$, thereby demolishing the lighter than $1 \mathrm{GeV}$ glueball of the MIT bag model.

Turning to the much heavier upsilon, the three gluons from its decay are expected to generate jet-like hadronic fragments. Normal electron-positron annihilation into hadrons by way of a quark-antiquark pair) above $6 \mathrm{GeV}$ produces two symmetrical jets opposite each other. This

\section{Polymers for blood replacement}

\section{from Paul Calvert}

SYNTHETIC blood volume expanders are sometimes used in cases of extensive bleeding or burns where much plasma has been lost, or in cases of shock, where contraction of the capillaries increases blood pressure so that water is lost to the tissues, the blood thickens and circulation ceases. Restoration of normal volume can be achieved by natural plasma, solutions of plasma proteins or by solutions of polymers such as dextran. Where blood or plasma are readily available the main reason for using a synthetic expander is to avoid the risk of hepatitis. On the other hand, in countries where blood supplies and storage and separation facilities are more limited, synthetic substitutes should be more important. So, recently published Chinese work on a volume expander derived from corn starch is of interest as an application of science to the needs of a developing country.

Blood has such diverse functions that it is difficult to imagine a completely satisfactory substitute. The role of a volume expander is simply to maintain the correct water balance between blood and the tissues and to allow continued transport of nutrients and gases. The water balance is determined by the osmotic pressure between blood and tissues, and the hydrostatic pressure difference. Lost volume can be restored by addition of an isotonic electrolyte solution such as Ringer's but this does not give the correct

Paul Calvert is a Lecturer in the School of Molecular Sciences, University of Sussex. jet structure survives the final 'colourwashing' that creates only colourless hadrons out of the quark and the antiquark. As each of the gluons from upsilon decay will on average carry an energy of nearly $3 \mathrm{GeV}$, gluon jets can be expected there. The three gluons should emerge symmetrically with $120^{\circ}$ angles between one another, and there is some preliminary evidence for such events from PLUTO (CERN Courier 19, 108; 1979). Another interesting possible configuration is one in which an energetic gluon dashes off to one side leaving two less energetic collinear ones opposite. This will lead to two jets opposite each other that are asymmetrical. According to a recent suggestion (Roy \& Walsh Phys. Lett. 78B, $62 ; 1978)$, the energetic gluon can now easily induce the creation of a gluon pair from the vacuum and capture one of its members to form a glueball. The latter should show up as a prominent, not very unstable resonance in one arm of the two jets and should disappear off the upsilon mass. Quantitative estimates, albeit handwaving, have been made and look extremely favourable for the production and detection of glueballs in this manner, if they exist at all.

osmotic pressure balance as the electrolytes pass readily across membranes into the tissues. It is necessary also to restore the colloidal osmotic (oncotic) pressure normally provided by serum albumin which cannot cross the membrane and so balances the osmotic pressure of the macromolecular cell contents. In practice it does not seem to be agreed whether polymeric (or 'colloidal') solutions work better than salt solutions but they are certainly better in principle (see Pennell, in Bibl. Haemat. 29, 883; 1968).

Solutions of a range of water-soluble polymers have been tried as volume expanders. The chief requirements are that the polymer is of sufficiently high molecular weight to prevent its loss through the kidneys, that it resists degradation and causes no harmful reactions. Dextran is chiefly used but hydroxyethyl starch has had successful clinical trials and polyvinyl pyrrolidone has been tested (Ricketts, Brit. J. Anaesth. $45,958 ; 1973)$. Dextran has been used for about 30 years and its side effects are well studied (Gruber \& Messmer, Prog. Surg. 15, 49; 1977); Gronwall et al., Bibl. Haemat. 29, 874; 1968). High molecular weight dextrans cause clumping of red cells (rouleaux formation) though, interestingly from the colloid science viewpoint, molecules of low molecular weight lower even the natural degree of clumping. Reduced clumping leads to lower blood viscosity, promotes blood flow and exchange of nutrients with tissues and so is beneficial in shock cases. High molecular weight dextran has anticoagulant activity and it can be used for this purpose after surgery. Low molecular weight dextran is antigenic but there is no evidence for this with clinically-used dextran, although allergic reactions occasionally occur. Various dextrans in the molecular weight range of $40,000-110,000$ are used depending on the balance of effect desired.

The Chinese work is from the Institute of Organic Chemistry in Shanghai, one of the three big polymer research groups in China. They describe the production of sodium carboxymethyl amylose by carboxymethylation of amylose from corn starch (Acta Chimica Sinica 36, 49 (1978). The molecular weight of the product was characterised by ultracentrifugation, solution viscometry, fractional precipitation and osmotic pressure. Using ${ }^{14} \mathrm{C}$-labelled carboxymethylation followed by hydrolysis and paper chromatography the reaction was shown to occur primarily on the 2-oxygen. The degradation rate of the polymer in blood decreases with increasing carboxymethylation. A 5\% solution of the polymer (molecular weight $60,000,0.4$ carboxyl methyl groups per saccharide) in $0.9 \%$ saline is now being tested in animals. It maintains blood volume for $6 \mathrm{~h}$, is $90 \%$ lost in $24 \mathrm{~h}$ and is said to lack the immunological reactions of dextran. A possible snag, however, is that similar charged polymers are reported to enhance clumping (see Rickets op.cit.)

This work is interesting in the Chinese context for a number of reasons. It is a piece of careful, thorough polymer science. It describes the development of a new material from an available resource to replace the complex fermentation process needed for dextran. The work is original, whereas much of polymer science in China has been devoted to reproducing Western materials from patents. Also the work was done between 1961 and 1964 and has waited 15 years to be published. It shows that a relatively modest project can produce something adapted to local needs.

Most Western interest in this area seems to be in the more dramatic problem of an artificial blood substitute capable of carrying oxygen, particularly for organ storage. Geyer (Drug Design VII (ed. Ariens) Academic Press, 1976), has reviewed these with particular regard to emulsions of perfluorochemicals. With these he was able to replace completely the blood of rats so that they survived in $100 \%$ oxygen for 10 days until their red cells regenerated and they could be returned to air. Interestingly, these bloodless rats could also survive in $10 \%$ carbon monoxide. Cell-free haemoglobin solutions have also been used for blood replacement but they are degraded and must be continually transfused. There is obviously potential here for an oxygenchelating polymer but a suitable molecule does not yet seem to be available 